\title{
Cosmic anisotropy with reduced relativistic gas
}

\author{
Simpliciano Castardelli dos Reis ${ }^{1, a}$, Ilya L. Shapiro ${ }^{1,2,3, b}$ \\ ${ }^{1}$ Departamento de Física, ICE, Universidade Federal de Juiz de Fora, Campus Universitário, Juiz de Fora, MG 36036-330, Brazil \\ 2 Tomsk State Pedagogical University, Tomsk 634041, Russia \\ ${ }^{3}$ Tomsk State University, Tomsk 634050, Russia
}

Received: 25 January 2018 / Accepted: 5 February 2018 / Published online: 20 February 2018

(C) The Author(s) 2018. This article is an open access publication

\begin{abstract}
The dynamics of cosmological anisotropies is investigated for Bianchi type I universe filled by a relativistic matter represented by the reduced relativistic gas model (RRG), with equation of state interpolating between radiation and matter. Previously it was shown that the interpolation is observed in the background cosmological solutions for homogeneous and isotropic universe and also for the linear cosmological perturbations. We extend the application of RRG to the Bianchi type I anisotropic model and find that the solutions evolve to the isotropic universe with the pressureless matter contents.
\end{abstract}

\section{Introduction}

The standard cosmological model describes a universe with homogeneous and isotropic geometry. The matter contents is described by a set of cosmic fluids satisfying some equations of state $(\mathrm{EoS})$. The inhomogeneities are allowed only in the form of small perturbations, which define most of the observables which are used to define most of relevant observables. We know that the spectrum of CMB, Large Scale Structure, $\mathrm{BAO}$ and other observations demonstrate the correctness of this description, that is the dynamics of perturbations proves that the expanding universe is very close to homogeneity and isotropy of expanding universe at the sufficiently large scale. The question is whether universe was "born" isotropic and homogeneous or it became such due to some internal mechanism at the early stage of its evolution. The complete analytical description of the possible anisotropies and nonhomogeneities of the early universe is impossible. Therefore the standard approach is to assume certain symmetry of the metric tensor. For instance, homogeneity and isotropy are possible symmetries. In formulating a more general metrics

\footnotetext{
a e-mail: simplim15@hotmail.com

be-mail: shapiro@ fisica.ufjf.br
}

one of the possibilities is to consider an anisotropic but homogeneous space-time. The pioneer work [1] explored the case of the metric anisotropic in two space directions with a given group of symmetries, in the universe filled by dust, while [2] dealt with the special cases of locally rotationally symmetric and shear-free dust.

The homogeneous models can be grouped by the possible space symmetries given by the Bianchi classification, which is based on the Lie algebras satisfied by the Killing vectors or, equivalently, the structure constants of the hypersurface's tetrad system [3]. The first possible space of this classification, called Bianchi type I, has three Killing vectors corresponding to the three spatial translations. Due to the simplicity of the Bianchi-I metric, it was extensively used in anisotropic cosmological models, including the Kasner vacuum solution [4]. More sophisticated cosmological models based on other types of Bianchi classification, are possible. One can mention, for instance, the renowed works on the Bianchi type IX models by Belinskii et al. [5,6], and the Mixmaster universe model by Misner [7,8], which shows a chaotic behaviour.

One of the main questions concerning anisotropic cosmological models is whether the universe could be anisotropic in the early epoch and evolve to be isotropic? It is certainly interesting to identify a mechanism which could be responsible by such an isotropization. It is highly desirable to have a maximally simple description of such a universe, such that further analysis of the perturbations could provide an observational evidence of isotropization. Some of mechanisms of this kind consist from the analysis of the asymptotic behaviour of solutions with isotropic classical fluids $[9,10]$ (see also [11]), viscous and anisotropic stress tensor [12], primordial magnetic field $[13,14]$ and quantum effects in primordial universe $[15,16]$.

In what follows we concentrate on the Bianchi-I model. From the mentioned references we know that the speed of isotropization of the metric may depend of the EoS of the 
contents of the universe and, in particular, is different for matter or radiation. The situation is qualitatively similar to the dynamics of metric and density perturbations on the isotropic background, but we do not need to treat anisotropies as small perturbations.

Usually, the EoS is assumed to be a linear relation between pressure and energy density, $p=\omega \rho$, with a constant $\omega$. The value of $\omega$ corresponds to the type of a fluid. For example, $\omega=-1$ means cosmological constant, $\omega=0$ dust and $\omega=1 / 3$ radiation. According to the recent data (see, e.g., $[17,18])$ the present-day universe is dominated by nonluminous sources, such as dark matter (DM) and dark energy. It is most likely that the DM is a gas of weakly interacting massive particles, while the main candidate to be dark energy is the cosmological constant. The observational data show that most of its history universe was very isotropic, and therefore the isotropization should occur very early. Since in the past the universe was much hotter than now, the contribution of the cosmological constant to the overall energy density balance at the epoch of isotropization was very small [19]. At the same time, regardless the mass and warmness of the DM particles are unknown, the DM is supposed to be very hot in the early universe and then to became relatively cold at the later stage. Therefore it makes sense to explore the isotropization mechanism for the case of a universe filled by baryonic and dark matter, which are hot in the early universe and dust-like in the present epoch. The simplest appropriate description for the particles in a very early universe is the ideal relativistic gas of massive particles. Perhaps the most useful representation of such a gas is through the reduced relativistic gas model (RRG), which provides a simplified approximation to the Maxwell distribution.

The EoS of RRG was originally invented by A.D. Sakharov in the famous 1966 paper [20], to interpolate between radiation and dust regimes. In this work the interpolating EoS has been used for the first derivation of the CMB spectrum, but the details of how to obtain the EoS of the model were not given. More recently RRG model was reinvented by our group in Refs. [21,22]. The main advantage of this model includes the fact that the solutions for the background cosmology can be obtained in a closed, analytic form for a wide class of models including RRG and other fluids [23], while the EoS is very close to the one of the relativistic gas of ideal particles [21]. Consequently RRG has been used for a simplified evaluation of the bounds of warmness of DM $[22,24]$, description of energy exchange between matter and radiation and for an overall rough estimate for the cosmological observables in the model with the running cosmological constant [25].

In the present work we apply RRG to describe the isotropization of the universe in the transition period when the matter contents of the universe is in the transition from the radiation to the dust EoS. We will follow the classical works $[9,13]$, but instead of dealing with radiation and dust cases separately, consider the RRG fluid which interpolates smoothly between the two regimes.

The paper is organized as follows. In Sect. 2 we present a new derivation of the EoS of the RRG [20]. This new derivation is instructive and more formal than the previous one in [21]. In Sect. 3 we formulate the equations describing the dynamics of Bianchi type I model in the universe filled by RRG. Section 4 describes the simplest approximation for solving these equations. In particular it is shown that the previously known radiation and dust cases represent the limiting cases of the new system of equations. The solution in the general case of RRG can be possible only by means of numerical methods, as described in Sect. 5. Finally, in Sect. 6 we draw our conclusions and describe the perspectives for the further work.

\section{Reduced relativistic gas: equation of state}

Let us consider the EoS for the RRG model in a way different from [21]. The model describes ideal relativistic gas of massive identical particles. The main simplification compared to the Jüttner model [26] (see also the book [27]) is that within RRG particles have identical kinetic energies. This assumption make the EoS very simple and, in particular, provides great simplification in cosmology, both at the background and perturbations level [20]. At the same time, the difference with the EoS of the Jüttner model, derived on the basis of Maxwell distribution does not exceed 2.5\% [21]. For the cosmological applications, since Jüttner model and, in general, an ideal gas of identical particles, is certainly just an approximation, the RRG is perfectly justified and useful model.

The derivation of EoS in [21] is very simple, one can say it is at the high-school level. Let us present a little bit more formal scheme of deriving this equation in the flat Minkowski metric, which enables one, in principle, to evaluate the difference with the Jüttner model analytically.

The number of particles $N$ is evaluated on a threedimensional space-like hypersurface with the normal vector $n^{\mu}$, with the hypersurface element area $d \sigma$. The general expression for a non-degenerate gas composted of identical particles is [28]

$N=\int d \sigma d^{4} p n_{\mu} p^{\mu} f(x, p) \delta\left(p^{2}-m^{2}\right)$,

where $p^{2}=\left(p^{0}\right)^{2}-\delta_{i j} p^{i} p^{j}$. The distribution function $f(x, p)$ depends of space-time coordinates and momenta, denoted by $x$ and $p$. Taking the integral over $d p_{0}$ and using the properties of the delta function, we get 
$N=\int d \sigma \frac{d^{3} p}{p^{0}} n_{\mu} p^{\mu} f(x, p)$.

For the constant time hypersurface $n^{\mu}=\delta_{0}^{\mu}$ and $d \sigma=d^{3} x$ we arrive at the expression

$N=\int d^{3} x d^{3} p f(x, p)$.

The RRG corresponds to the ansatz for distribution function,

$f(x, p)=C \delta\left(E-E_{0}\right)$,

where $C$ is a normalization constant, $E=p^{0}=\sqrt{\mathbf{p}^{2}+m^{2}}$ and $E_{0}$ is a constant energy of a gas particle. Using the expression for distribution function in (3), one can easily obtain

$N=C \int d^{3} x d \Omega d E E \sqrt{E^{2}-m^{2}} \delta\left(E-E_{0}\right)$,

where $d \Omega$ is the solid angle element. From the last expression, one can determine the constant $\mathrm{C}$, leading to the final form of the distribution function,

$f=\frac{n}{4 \pi E_{0} \sqrt{E_{0}^{2}-m^{2}}} \delta\left(E-E_{0}\right)$,

Here $n=N / V$ is the concentration (number of particles per volume) of the gas. The expression for the energy-momentum tensor is $[3,28]$ (see also brief derivation in the Appendix)

$T^{\mu \nu}=\int d^{3} p \frac{p^{\mu} p^{\nu}}{p^{0}} f(x, p)$.

In the reference frame of an observer with four-velocity $u^{\mu}$ the projection of the energy-momentum tensor onto the hypersurface with normal vector $u^{\mu}$ leads to the energy density $\rho$ and pressure $p$. According to Ref. [11],

$\rho=u_{\mu} u_{\nu} T^{\mu \nu}, \quad p=-\frac{1}{3} h_{\mu \nu} T^{\mu \nu}$,

where $h_{\mu \nu}=\eta_{\mu \nu}-u_{\mu} u_{\nu}$. In case of a comoving reference frame, in which observer has the four-velocity $u^{\mu}=\delta_{0}^{\mu}$ with the distribution function (6), the expressions (8) become

$\rho=n E_{0} \quad$ and $\quad p=\frac{n\left(E_{0}^{2}-m^{2}\right)}{3 E_{0}}$.

Defining the rest energy density $\rho_{d}=n m$, the pressure and energy density are related by the expression

$p=\frac{\rho}{3}\left(1-\frac{\rho_{d}^{2}}{\rho^{2}}\right)$,

which is nothing else but the EoS of the RRG model [20,21]. It is easy to see that this EoS interpolates between radiation, $p \sim \rho / 3$, at high energies, when $\rho^{2} \gg \rho_{d}^{2}$, and dust $p \sim 0$, at low energies, when $\rho^{2} \approx \rho_{d}^{2}$.

\section{Bianchi-I type cosmology with RRG}

Consider the anisotropic cosmology with the RRG fluid. Our starting point will be the space of Bianchi-I type, with the metric of the form [3],

$d s^{2}=d t^{2}-a_{1}^{2}(t) d x^{2}-a_{2}^{2}(t) d y^{2}-a_{3}^{2}(t) d z^{2}$.

A useful parametrization of anisotropic metric was introduced by Misner in [7,8],

$a_{1 / 2}(t)=a(t) e^{\beta_{+}(t) \pm \sqrt{3} \beta_{-}(t)}, \quad a_{3}(t)=a(t) e^{-2 \beta_{+}(t)}$,

where $a(t), \beta_{+}(t)$ and $\beta_{-}(t)$ are unknown functions of time. In this parametrization $\sqrt{-g}=a_{1} a_{2} a_{3}=a^{3}$ and the relation between $\beta_{ \pm}$and $a_{i}$ is

$\beta_{+}=\frac{1}{6} \ln \left(\frac{a_{1} a_{2}}{a_{3}^{2}}\right) \quad \beta_{-}=\frac{1}{2 \sqrt{3}} \ln \left(\frac{a_{1}}{a_{2}}\right)$.

In Ref. [11], within the $1+3$ covariant formalism of a system of time-like geodesic congruence, the change of a connecting vector between geodesics, expressing the relative distance, is split in the irreducible parts called shear, vorticity and expansion. In particular, the functions $\beta_{ \pm}$are the independent components of a traceless tensor, which represents the shear. In what follows, we analyse the dynamics of gravitational field for the metric (12), generated by Einstein equations. The matter contents of the universe is modelled by an isotropic $\mathrm{RRG}$, where pressure is assumed to be the same in all spatial directions.

The energy-momentum tensor is

$T_{\mu \nu}=(\rho+p) u_{\mu} u_{\nu}+p g_{\mu \nu}$.

The conservation equation $\nabla_{\mu} T^{\mu \nu}=0$ leads to

$\dot{\rho}+3 H(\rho+p)=0, \quad H=\frac{\dot{a}}{a}$,

where the dot means derivative with respect to the physical time. Let us note that the anisotropy of the metric does not affect the last equation because of the isotropic pressure. Equation (15) can be integrated by using the EoS of the RRG, yielding the same result as in the isotropic case [21],

$\rho=\sqrt{\rho_{1}^{2}\left(\frac{a_{0}}{a}\right)^{6}+\rho_{2}^{2}\left(\frac{a_{0}}{a}\right)^{8}}$,

where $\rho_{1}, \rho_{2}$ and $a_{0}$ are integration constants.

As usual, one can distinguish two extreme regimes in the solution (16). In the case $\rho_{1} \ll \rho_{2}$, one meets the ultrarelativistic case, that is RRG demonstrates radiation-like behaviour. On the other hand, for $\rho_{1} \gg \rho_{2}$, RRG behaves like a dust.

Now we are in a position to consider the Einstein equations for the Bianchi-I metric. According to Ref. [12], the Einstein 
tensor, $G_{\mu v}$ for the metric (12) assumes the form

$$
\begin{aligned}
G_{00}= & 3 H^{2}-3\left(\dot{\beta}_{+}^{2}+\dot{\beta}_{-}^{2}\right), \\
G_{11}= & -3 H^{2}-2 \dot{H}-3\left(\dot{\beta}_{+}^{2}+\dot{\beta}_{-}^{2}\right) \\
& +\left(\frac{d^{2}}{d t^{2}}+3 H \frac{d}{d t}\right)\left(\beta_{+}+\sqrt{3} \beta_{-}\right), \\
G_{22}= & -3 H^{2}-2 \dot{H}-3\left(\dot{\beta}_{+}^{2}+\dot{\beta}_{-}^{2}\right) \\
& +\left(\frac{d^{2}}{d t^{2}}+3 H \frac{d}{d t}\right)\left(\beta_{+}-\sqrt{3} \beta_{-}\right), \\
G_{33}= & -3 H^{2}-2 \dot{H}-3\left(\dot{\beta}_{+}^{2}+\dot{\beta}_{-}^{2}\right) \\
& -2\left(\frac{d^{2}}{d t^{2}}+3 H \frac{d}{d t}\right) \beta_{+} .
\end{aligned}
$$

The Einstein equations are given by $G_{\mu \nu}=8 \pi G T_{\mu \nu}$. For an isotropic $T_{\mu \nu}$ tensor, Einstein equations can be rewritten such that the pressure of matter does not enter the equations. Following [12], we define the new quantities

$$
\begin{aligned}
& G_{+}=\frac{1}{6}\left(G_{11}+G_{22}-2 G_{33}\right), \\
& G_{-}=\frac{1}{2 \sqrt{3}}\left(G_{11}-G_{22}\right),
\end{aligned}
$$

yielding significant simplifications compared to (17),

$G_{ \pm}=\ddot{\beta}_{ \pm}+3 H \dot{\beta}_{ \pm}$.

Einstein equations boil down to ${ }^{1}$

$G_{+}=\frac{8 \pi G}{6}\left(T_{11}+T_{22}-2 T_{33}\right)$,

$G_{-}=\frac{8 \pi G}{2 \sqrt{3}}\left(T_{11}-T_{22}\right)$

Furthermore, since we assume isotropic energy-momentum tensor, $T_{11}=T_{22}=T_{33}$ and

$G_{ \pm}=0$.

Finally, 00-component of Einstein equations, together with Eqs. (19) and (21), yield

$$
H^{2}-\left(\dot{\beta}_{+}^{2}+\dot{\beta}_{-}^{2}\right)=\frac{8 \pi G}{3} \rho
$$

and $3 H \dot{\beta_{ \pm}}+\ddot{\beta_{ \pm}}=0$.

A first integral of (23) can be easily found in the form

$\dot{\beta_{ \pm}}=\gamma_{ \pm} a^{-3}$

where $\gamma_{ \pm}$are integration constants. The last result transforms (22) into an equation for the conformal factor of isotropic expansion $a(t)$. Defining useful constants $\Gamma$ and $\phi$,

$\gamma_{+}=\Gamma \cos \phi, \quad \gamma_{-}=\Gamma \sin \phi$

$\overline{1 \text { Equation (20) }}$ also follows from the variation of the Einstein-Hilbert action with respect to $\beta_{ \pm}$. we arrive at the generalized form of Friedmann equation for anisotropic Bianchi-I metric with RRG matter contents,

$$
\begin{aligned}
H^{2}=\frac{\dot{a}^{2}}{a^{2}}= & \Gamma^{2}\left(\frac{a_{0}}{a}\right)^{6}+\frac{8 \pi G}{3} \rho_{1}\left(\frac{a_{0}}{a}\right)^{4} \\
& \times \sqrt{\left(\frac{a_{0}}{a}\right)^{-2}+b^{2}},
\end{aligned}
$$

where $b=\rho_{2} / \rho_{1}$ is the warmness parameter [22]. The specific new element compared to isotropic cosmological model is the first term in the r.h.s.. This term has the ultrarelativistic $a^{-6}$ scaling feature and hence it is irrelevant for the late cosmology. At the same time, it may be quite relevant in the early universe. Due the new term, caused by anisotropy, the very early universe behaves according to

$a \sim t^{1 / 3}$

different from the radiation-dominated universe. It is wellknown that the same dynamics of the conformal factor can be achieved in the isotropic plane universe with an ideal fluid with $\operatorname{EoS} p=\rho$. In order to see this consider the $\operatorname{EoS} p=$ $w \rho$, with constant $w$. Using the conservation law results in $\rho=\rho_{0} a^{-3(w+1)}$. By comparing this result to the first term of the r.h.s of (26), we arrive at $w=1$. A fluid of this kind was called stiff matter, when first introduced by Zel'dovich [29]. We have seen that this EoS results from integrating anisotropies at the early stage of the evolution of the universe.

The solution of Eq. (24) can be expressed as

$\beta_{ \pm}(t)-\beta_{ \pm}^{0}=\gamma_{ \pm} W(t), \quad W(t)=\int_{t_{0}}^{t} \frac{d t^{\prime}}{a^{3}\left(t^{\prime}\right)}$.

In this expression $t_{0}$ correspond to the initial moment of time and $\beta_{ \pm}^{0}$ are integration constants. One can notice that both $\beta_{ \pm}$, with exception of the integration constants, will lead to a same functional form. After $W(t)$ is found, the parameters $\gamma_{ \pm}$determine $\beta_{ \pm}$and consequently the metric components by Eq. (12).

\section{Approximations}

It is easy to present the solution for (26) and (28) in the form of quadratures, however the integrals are not elementary functions and the qualitative analysis becomes cumbersome. Therefore, in order to have better idea about the physical output of these equations, we split the derivation of the scale factor dependence into two different considerations. In the present section we consider three approximations, namely, vacuum, radiation and dust. In the next section we present the results of a numerical solution in the general case. The radiation and dust approximations come from the limits of the RRG EoS depending on the value of parameter $b$. The approximation for vacuum will be explained bellow. The considerations in this section are almost completely non-original 
and are presented as to serve as reference for the consequent numerical solutions. When $a(t)$ is very small, one can keep only the first (stiff matter of anisotropic origin) term on the r.h.s of (26). This procedure is equivalent to taking a vacuum solution, because in this regime we are disregarding the terms coming from the matter contents. Indeed, it is known that for the evolution of homogeneous and anisotropic models in the vicinity of the singularity the matter contents has no much relevance [30] (see also [31]).

The vacuum metric of Bianchi type I is called Kasner solution. Following [12], we arrive at

$\frac{\dot{a}^{2}}{a^{2}}=\Gamma^{2}\left(\frac{a_{0}}{a}\right)^{6}$,

which can be solved in the form

$\left(\frac{a}{a_{0}}\right)^{3}=3 \Gamma\left(t-t_{0}\right)$.

Setting $a_{0}=1$, the Eq. (28) can be integrated, yielding

$\beta_{ \pm}(t)=\beta_{ \pm}^{(0)}+\frac{\gamma_{ \pm}}{3 \Gamma} \ln \left(\frac{t}{t_{0}}\right)$.

Here $\beta_{ \pm}^{(0)}$ and $t_{0}$ are integration constants. From the angular relations (25) the functions $a_{k}(t)$ can be presented as

$a_{k}(t)=(3 \Gamma)^{1 / 3} t^{p_{k}}, \quad k=1,2,3$.

Parameters $p_{k}$ can be written down using notations (25),

$$
\begin{aligned}
p_{1 / 2} & =\frac{1}{3}(1+\cos \phi \pm \sqrt{3} \sin \phi), \\
p_{3} & =\frac{1}{3}(1-2 \cos \phi) .
\end{aligned}
$$

and the line element as

$d s^{2}=d t^{2}-(3 \Gamma)^{2 / 3}\left[t^{2 p_{1}} d x^{2}-t^{2 p_{2}} d y^{2}-t^{2 p_{3}} d z^{2}\right]$,

The multiplicative constant can be absorbed into the spatial coordinates, providing the standard form [3],

$d s^{2}=d t^{2}-t^{2 p_{1}} d x^{2}-t^{2 p_{2}} d y^{2}-t^{2 p_{3}} d z^{2}$,

where the parameters $p_{k}, p_{2}$ and $p_{3}$ satisfy the algebraic constraints

$p_{1}^{2}+p_{2}^{2}+p_{3}^{2}=1, \quad p_{1}+p_{2}+p_{3}=1$.

Finally, in the Kasner solution

$a(t)=\left[a_{1}(t) a_{2}(t) a_{3}(t)\right]^{1 / 3}=t^{\frac{1}{3}\left(p_{1}+p_{2}+p_{3}\right)}=t^{1 / 3}$.

The approximations for which the analytic solution can be easily obtained correspond to the ultra-relativistic, $b^{-1} \rightarrow 0$ or dust, $b \rightarrow 0$ regimes. In what follows we consider these two cases separately. Let us note that the general form of solutions (28) remains the same independent on the approximations for the isotropic energy-momentum tensor.

In the ultra-relativistic case one can perform the expansion up to the first order in $b^{-1}$ in (26). Taking $a_{0}=1$, we arrive at

$\dot{a}^{2}=\frac{\Gamma^{2}}{a^{4}}+\frac{8 \pi G \rho_{1} b}{3 a^{2}}\left(1+\frac{a^{2}}{2 b^{2}}\right)$.

Taking into account the $a^{2} / b^{2}$-term in the parenthesis, this is the Bianchi type I model with radiation, which has initial density expressed by $\rho_{2}$. This is exactly the classical result of [9] for the radiation, but we obtained it as a limit of the RRG solution.

It proves useful to make a change of variables

$a=\frac{1}{\chi_{\text {rad }}} \sinh \xi, \quad \kappa_{\mathrm{rad}}^{2}=\frac{8 \pi G \rho_{2}}{3 \Gamma^{2}}, \quad 0 \leq \xi<\infty$,

in Eq. (26). This results in the relation

$d t=\frac{1}{\Gamma \kappa_{\mathrm{rad}}^{3}} \sinh ^{2} \xi d \xi$.

Then Eqs. (26) and (28) become the parametric relations

$\Gamma t=\frac{1}{4 \kappa_{\mathrm{rad}}^{3}}(\sinh 2 \xi-2 \xi), \quad \beta_{ \pm}=\frac{\Gamma_{ \pm}}{\Gamma} \ln (\tanh \xi)$.

From the relation between $a$ and $\xi$ in (39), one can obtain

$\tanh \xi=\left(1+\frac{1}{a^{2} \kappa_{\text {rad }}^{2}}\right)^{-\frac{1}{2}}$.

In case $\left(a^{2} \kappa_{\text {rad }}^{2}\right)^{-1}$ is very small, one gets the relation

$\tanh \xi=1-\frac{1}{2} \frac{1}{a^{2} \kappa_{\mathrm{rad}}^{2}}+\cdots$.

Consequently, due to the (41),

$\beta_{ \pm}=-\frac{\gamma_{ \pm}}{\Gamma}\left[\frac{1}{a^{2} \kappa_{\mathrm{rad}}^{2}}+\cdots\right]$.

In the radiation approximation, if we disregard the term $\left(a^{2} \kappa_{\text {rad }}^{2}\right)^{-1}$, then $\beta_{ \pm}$tend to zero for great values of $a$, and effectively there is isotropization.

Another way to arrive at the same conclusion is by observing that when $\xi \rightarrow \infty$, we have $\beta_{ \pm} \rightarrow 0$. In the same limit

$\sinh \xi \sim \cosh \xi \sim \frac{1}{2} e^{\xi}$

and dominate in the Eq. (41). Using (39),

$t \approx \frac{2 a^{2}}{\kappa_{\mathrm{rad}}}$

which yields the standard expression for the isotropic radiation dominated universe,

$a=\left(\frac{2 \pi G \rho_{2}}{3 \Gamma^{2}}\right)^{1 / 4} \sqrt{t}$. 
This expression means that the role of anisotropy is negligible for the evolution of the scale factor and hence we have isotropization.

Let us now consider the limit $a \gg b$, which means a dustdominated universe. The solution of the dynamical Eqs. (26) and (28) for dust is simpler than for the radiation-dominated case [12] and was originally obtained in [32]. Here we will try to arrive at the same result by taking the corresponding limit in the general solution for RRG, which interpolates between radiation and dust.

The solutions of Eqs. $(26,28)$ for $a(t)$ and $\beta_{ \pm}(t)$ are given by

$a^{3}=\frac{3 \Gamma}{t_{I}} t\left(t+t_{I}\right), \quad \beta_{ \pm}=\frac{\gamma_{ \pm}}{3 \Gamma} \ln \left(\frac{t}{t+t_{I}}\right)$.

Here

$t_{I}=\frac{4}{3 \Gamma \kappa_{\mathrm{dust}}^{2}} \quad$ and $\quad \kappa_{\mathrm{dust}}^{2}=\frac{8 \pi G \rho_{1}}{3 \Gamma^{2}}$

are constants. The solutions (48) in the dust-dominated approximation can be considered in two different asymptotic situations. The first one is $t \ll t_{I}$, which implicates in the expansions

$a^{3}=3 \Gamma\left[\left(\frac{t}{t_{I}}\right)^{2}+t\right]$,

$\beta_{ \pm}=\frac{\gamma_{ \pm}}{3 \Gamma} \ln \left[\frac{t}{t_{I}}\left(1+\frac{t}{t_{I}}+\cdots\right)\right]$.

Disregarding terms with powers greater than two, the solutions tend to the Kasner expressions (30) and (31).

In the second case $t \gg t_{I}$ one can use the same scheme as before, but now making expansion in the powers of $t_{I} / t$. In this way we obtain the standard solution for the dust, with $a \sim t^{2 / 3}$ and $\beta_{ \pm} \rightarrow 0$. Following the same logic as in the radiation case, we conclude that the behaviour in the late times demonstrates isotropization.

\section{Numerical solution}

Let us consider numerical solution of the dynamical system of Eqs. (26) and (28) without assuming high- or low-energy approximations. Exactly as it was done in the previous section, we consider a simplified model with one fluid described by RRG and the anisotropy which enters the general energy balance by means of the stiff matter energy density. It proves useful to express the solution in terms of initial values of the relative energy densities parameters $\Omega_{a n}^{(i)}$ and $\Omega_{R R G}^{(i)}$, defined by

$\Omega_{a n}=\frac{\Gamma^{2}}{H^{2}}, \quad \Omega_{R R G}=\frac{8 \pi G \rho_{1}}{3 H^{2}} \sqrt{1+b^{2}}=1-\Omega_{a n}$.
The subscript $(i)$ denotes the values of the parameters in the initial moment of time. Our purpose is to evaluate the isotropization of the universe starting from the initial moment of time $t=0$, when $a(0)=a_{i}=1$ and $H(0)=H_{i}$. Therefore, in the initial instant of time the values are $\Omega_{a n}^{(i)}$ and $\Omega_{R R G}^{(i)}$, corresponding to $H=H_{i}$ in (51).

It proves useful to define the dimensionless time variable $\tau=H_{i} t$. In this way we arrive in the equations

$\frac{\dot{a}^{2}}{a^{2}}=\frac{\Omega_{a n}^{(i)}}{a^{6}}+\frac{\Omega_{R R G}^{(i)}}{a^{4} \sqrt{1+b^{2}}} \sqrt{a^{2}+b^{2}}$,

$\dot{\beta}_{ \pm}=\frac{\sqrt{\Omega_{a n}^{(i)}} \gamma_{ \pm}}{\Gamma a^{3}}$,

where the dots mean derivatives with respect to $\tau$,

The value of $\Omega_{a n}(t)$ measures the amount of anisotropy, such that greater values correspond to higher degree of anisotropy. As before, $b$ is the warmness parameter of the RRG matter. In the nowadays universe the value of $\Gamma$ is very small implying in a very small value of $\Omega_{a n}^{0}$. The warmness $b$ today is bounded from above by approximately 0.001 for the dominating fluid, namely for the dark matter [25]. Indeed, in the early universe when $\Omega_{a n}^{0}$ was significant, the warmness could have a large value. The framework of RRG enables one to see how the warmness affects the time of isotropization, that is the typical time of transition from large value of $\Omega_{a n}^{(i)}$ to a small value at the later period.

The second equation in (52) can be expressed via the angular parameter in (25). This angle becomes relevant only in the vicinity of the singularity, when the metric can be approximated by the Kasner solution, and in the subsequent numerical analysis it will not play much role.

Let us present the numerical solutions for different values of the warmness parameter $b$ using Mathematica software [33]. We used the initial conditions $a=a_{i}=1, \beta_{+}=10$, $\beta_{-}=15$, such that $\Omega_{a n}^{(i)}=0.99$ and $\Omega_{R R G}^{(i)}=0.01$ at $\tau=0$. In all plots the scale factor and anisotropy measure $\Omega_{a n}$ are compared with the plots for the cases of vacuum, radiation and dust, by assuming the same initial values of $\Omega^{(i)}$ 's. The Figs. 1 and 2 clearly shows that RRG behaviour tends to Kasner at the early stage of evolution, and is very close of radiation during some time for both scale factor and $\Omega_{a n}(\tau)$. In the Figs. 3 and 4 , the isotropisation can be observed, because $\beta_{+}$and $\beta_{-}$tend to constants. It is easy to see that the isotropization for RRG occurs faster than for the dust-like contents, close to the rate in the radiation case.

For smaller warmness, $b=0.5$, one can observe in Figs. 5, 6, 7 and 8 another behaviour, when RRG plot is (quite naturally) close to dust.

The plots presented above show that the RRG is perfectly well interpolating between radiation and dust regimes, as it should be expected. The asymptotic behavior of $\beta_{ \pm}(\tau)$ is constant, which means an effective isotropization of the solu- 


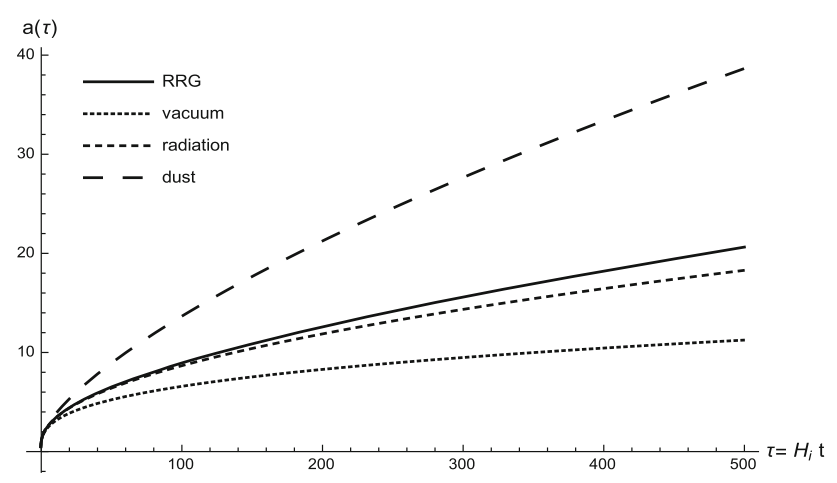

Fig. 1 Plots of scale factors for the initial values of $b=10$ and $\Omega_{a n i}^{(i)}=$ 0.99

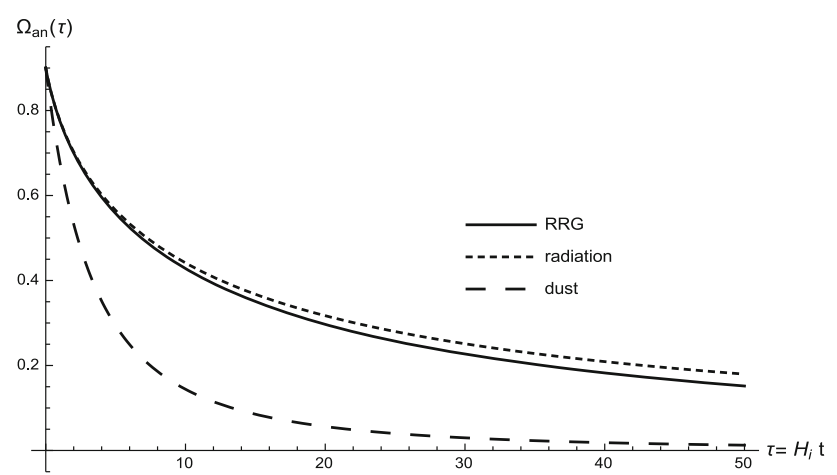

Fig. 2 Plots of $\Omega_{a n i}(\tau)$ for the initial values of $b=10$ and $\Omega_{a n i}^{(i)}=$ 0.99

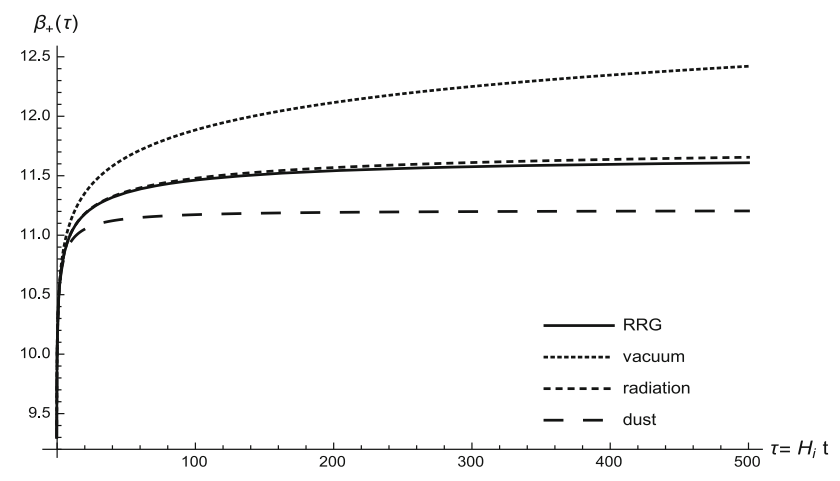

Fig. 3 Plots of $\beta_{+}$corresponding to the parameters $b=10$ and $\Omega_{a n i}^{(i)}=$ 0.99 , while the initial condition $\beta_{+}=10$

tions. Concerning the time of isotropization, depending on warmness the RRG model can be closer to dust or radiation.

\section{Conclusions}

We formulated the framework of RRG model applied to the dynamics of anisotropy in the early epoch, where the universe was filled by radiation and matter (baryonic and dark), which was so hot that has the EoS which interpolates between the radiation and pressureless matter. For the Bianchi-I universe away from the singularity region the gravitational theory

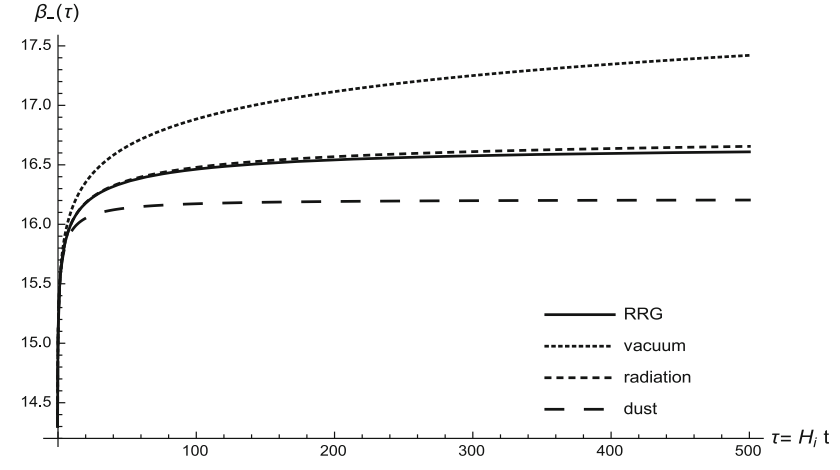

Fig. 4 Plots of $\beta_{-}$corresponding to the parameters $b=10$ and $\Omega_{a n i}^{(i)}=$ 0.99 , while the initial condition $\beta_{-}=15$

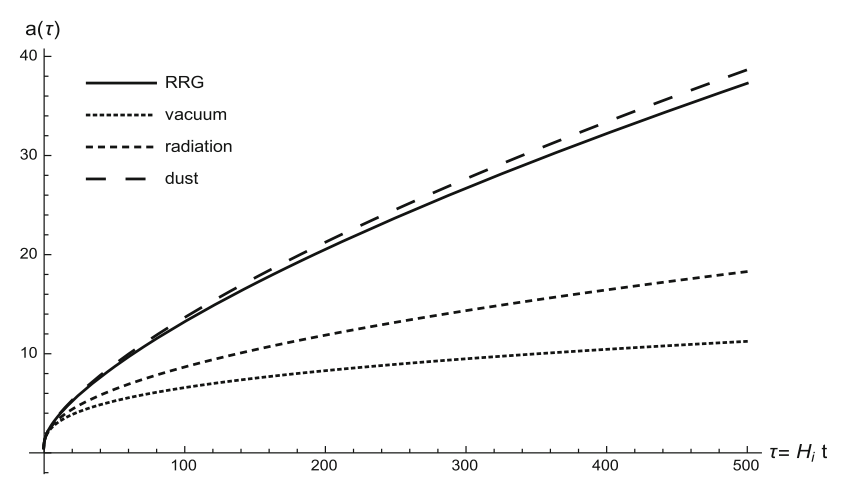

Fig. 5 Plots of scale factors for the moderate warmness. Parameters are as follows: $b=0.5, \Omega_{a n i}^{(i)}=0.99$

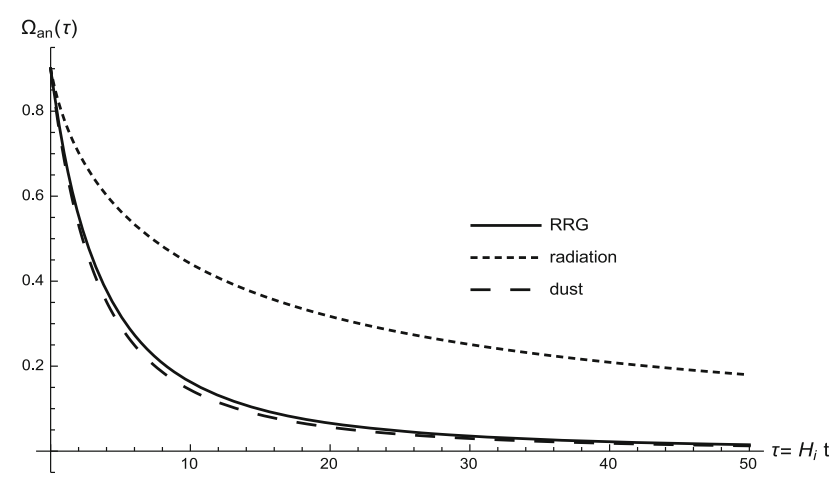

Fig. 6 Plots of $\Omega_{a n i}(\tau)$ for the moderate warmness. Parameters are as follows: $b=0.5, \Omega_{a n i}^{(i)}=0.99$

based on the Einstein-Hilbert action provides an isotropization mechanism for RRG, exactly like for both radiation and dust matter contents with isotropic EoS. This physical situation is a subject of current interest, see, e.g., [34]. More complicated spaces may require a more complicated gravitational theories to explain isotropization mechanism. We believe that the simple and efficient RRG model can be useful for describing the realistic matter contents in these complicated cases, as it was for the rather simples Bianchi-I universe described above. 


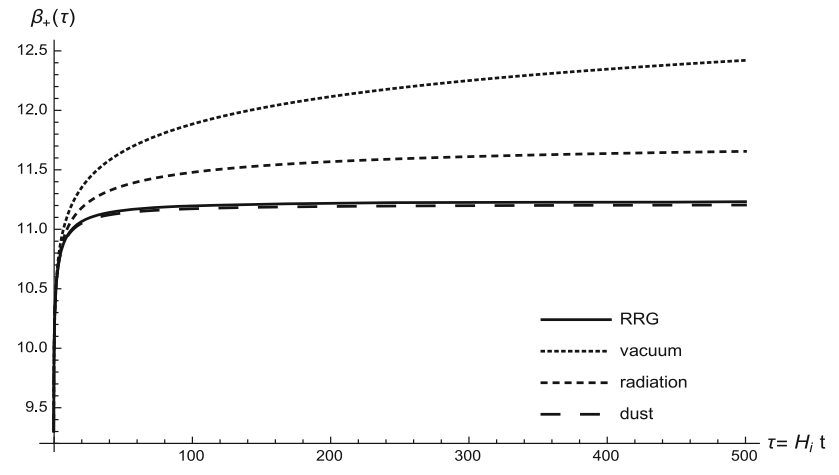

Fig. 7 Plots of $\beta_{+}$for the moderate warmness. Parameters are as follows: $b=0.5, \Omega_{a n i}^{(i)}=0.99$ with the initial condition $\beta_{+}=10$

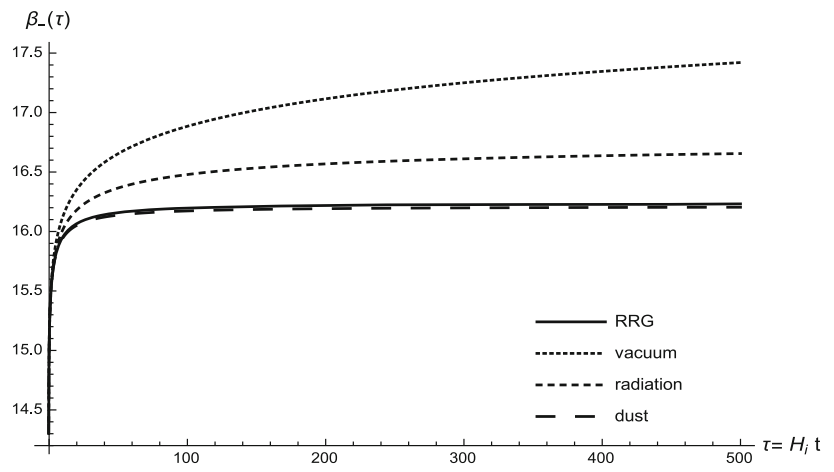

Fig. 8 Plots of $\beta_{-}$for the moderate warmness. Parameters are as follows: $b=0.5, \Omega_{\text {ani }}^{(i)}=0.99$ while the initial condition is $\beta_{-}=15$

Another potentially interesting application of our results is related to the cosmic perturbations in the anisotropic universe, which is not sufficiently well explored. Since the problem is technically complicated, it maybe very useful to have a simple albeit realistic description of the matter contents in the early universe in the epoch when the isotropization occurs. In this respect the framework RRG looks perfect, since it is extremely simple and enables one to quantify the transition from radiation to matter epochs, exactly as it was used in the pioneer work of Sakharov [20].

As it was expected from the previous works on this model $[21,22,25]$, the RRG shows the behaviour which is intermediate between radiation and dust and approaches one or another depending on the value of warmness parameter. We have shown that this feature can be extended to the simplest anisotropic Bianchi-I model.

One of the natural further developments can be related to the derivation and analysis of density and metric perturbations in the universe filled by RRG, including the case with interaction between RRG and radiation [35]. One can expect that RRG would be eventually useful as a model which helps to explore the observables which can tell us about the dynamics of anisotropies in the early universe.

The formalism which was developed in the present work can be useful for the description of Bianchi I phase between the two FLRW phases of the history of universe in the models proposed in $[36,37]$. In this case the matter contents of the universe is supposed to be hot and therefore the RRG can be helpful in its efficient description.

One can also use the same description of the hot or warm matter in other approaches to anisotropy, like the recent consideration of gravity with $R^{2}$ term [38] or with the sigmamodel like scalar field [39], or even in loop quantum gravity [40].

Acknowledgements Authors are very grateful to Patrick Peter for useful discussions. SCR is grateful to CAPES for supporting his Ph.D. project. IS was partially supported by CNPq, FAPEMIG and ICTP.

Open Access This article is distributed under the terms of the Creative Commons Attribution 4.0 International License (http://creativecomm ons.org/licenses/by/4.0/), which permits unrestricted use, distribution, and reproduction in any medium, provided you give appropriate credit to the original author(s) and the source, provide a link to the Creative Commons license, and indicate if changes were made. Funded by SCOAP $^{3}$.

\section{Appendix. Brief derivation of Eq. (7)}

Let us present a very brief derivation of the main expression for the energy-momentum tensor which was used in the main text to arrive at the EoS of the RRG model. More details can be found in [28] and also in [3].

Consider a gas of free massive relativistic particles with equal masses in the equilibrium state. Once in the comoving frame for each particle $T^{00}$ is the energy density, the standard arguments show that the energy-momentum tensor of the gas can be expressed as a sum over particles which are labeled by the subscript $a$,

$T^{\mu \nu}(x)=\sum_{a} \int d s \delta^{4}\left(x-x_{a}(s)\right) \frac{p_{a}^{\mu}(s) p_{a}^{v}(s)}{m_{a}}$,

and $s$ is an integration over the proper time for individual particles. Using the definition of Dirac's delta function, one can rewrite this expression in the form

$T^{\mu v}(x)=\int d^{4} p p^{\mu} p^{v} f(x, p)$,

where

$f(x, p)=\sum_{a} \int d s \frac{\delta^{4}\left(p-p_{a}(s)\right) \delta^{4}\left(x-x_{a}(s)\right)}{m_{a}}$.

The expression (54) includes an integral over four-momenta. As far as each of the free particles satisfies a dispersion relation $p^{2}=m^{2}$ with $p^{0} \geq 0$, one can replace $d^{4} p$ by the expression

$d^{3} p d p^{0} \delta\left(p_{0}^{2}-\mathbf{p}^{2}-m^{2}\right)$. 
Taking the integral over $p^{0}$ one has to replace the invariant element of integration in four dimensions $d^{4} p$ to the invariant element of integration in the space sector, $\left(m / p^{0}\right) d^{3} p$, because the normal vector to the $p^{2}=m^{2}$ has the same direction as $p^{\mu}$ [3]. Finally, using the properties of the delta function leads us to Eq. (7), where the distribution function $f$ depends only on $\mathbf{p}$.

Let us stress that the distribution function $f(x, p)$ is defined to be dependent on the motion of all particles. For the many-body system the use of the methods of Statistical Mechanics, in the case of a thermal equilibrium in Minkowski space leads to the distribution function of the Jüttner model. The simplifying assumption of the RRG is that all particles have the same kinetic energy, and that is why the distribution function is chosen as a delta function. As we know from the previous work [21], this approach provides an excellent approximation to complicated EoS of the Jüttner model.

\section{References}

1. R. Kantowski, R.K. Sachs, Some spatially homogeneous anisotropic relativistic cosmological models. J. Math. Phys. 7, 443 (1966)

2. G.F.R. Ellis, Dynamics of pressure free matter in general relativity. J. Math. Phys. 8, 1171 (1967)

3. L.D. Landau, E.M. Lifshitz, The Classical Theory of Fields (Butterworth Heinemann, New York, 1987)

4. E. Kasner, Geometrical theorems on Einstein's cosmological equations. Am. J. Math. 43, 217.0 (1921)

5. V.A. Belinsky, I.M. Khalatnikov, E.M. Lifshitz, Oscillatory approach to a singular point in the relativistic cosmology. Adv. Phys. 19, 525 (1970)

6. V.A. Belinsky, I.M. Khalatnikov, E.M. Lifshitz, A general solution of the Einstein equations with a time singularity. Adv. Phys. 31, 639 (1982)

7. C.W. Misner, The isotropy of the universe. Astrophys. J. 151, 431 (1968)

8. C.W. Misner, Mixmaster universe. Phys. Rev. Lett. 22, 1071 (1969)

9. K.C. Jacobs, Bianchi type I cosmological models. Thesis

10. G.F.R. Ellis, M.A.H. MacCallum, A class of homogeneous cosmological models. Commun. Math. Phys. 12, 108 (1969)

11. G.F.R. Ellis, R. Maartens, M.A.H. MacCallum, Relativistic Cosmology (Cambridge University Press, Cambridge, 2012)

12. O. Groen, S. Hervik, Einstein's General Theory of Relativity: With Modern Applications in Cosmology (Springer, New York, 2007)

13. K.C. Jacobs, Cosmologies of Bianchi type I with a uniform magnetic field. Astrophys. J. 155, 379 (1969)

14. K.S. Thorne, Primordial element formation, primordial magnetic fields, and the isotropy of the universe. Astrophys. J. 148, 51 (1967)

15. V.N. Lukash, I.D. Novikov, A.A. Starobinsky, Y.B. Zeldovich, Quantum effects and evolution of cosmological models. Nuovo Cim. B 35, 293 (1976)

16. B.L. Hu, L. Parker, Anisotropy damping through quantum effects in the early universe. Phys. Rev. D 17, 933 (1978). (Erratum: [Phys. Rev. D 17, 3292 (1978)])

17. A.G. Riess et al. [Supernova Search Team], Observational evidence from supernovae for an accelerating universe and a cosmological constant. Astron. J. 116, 1009 (1998). arXiv:astro-ph/9805201
18. L. Bergström, Nonbaryonic dark matter: observational evidence and detection methods. Rep. Prog. Phys. 63, 793 (2000). hep$\mathrm{ph} / 0002126$

19. S.A. Bludman, M.A. Ruderman, Induced cosmological constant expected above the phase transition restoring the broken symmetry. Phys. Rev. Lett. 38, 255 (1977)

20. A.D. Sakharov, The initial stage of an expanding universe and the appearance of a nonuniform distribution of matter. Sov. Phys. JETP 22, 241 (1966) [Zh. Eksp. Teor. Fiz. 49(1), 345 (1966)]

21. G. de Berredo-Peixoto, I.L. Shapiro, F. Sobreira, Simple cosmological model with relativistic gas. Mod. Phys. Lett. A 20, 2723 (2005). arXiv:gr-qc/0412050

22. J.C. Fabris, I.L. Shapiro, F. Sobreira, DM particles: how warm they can be? JCAP 0902, 001 (2009). arXiv:0806.1969

23. L.G. Medeiros, Cosmological analytic solutions with reduced relativistic gas. Mod. Phys. Lett. A 27, 1250194 (2012). arXiv: 1209.4930

24. W.S. Hipolito-Ricaldi, R.F. vom Marttens, J.C. Fabris, I.L. Shapiro, L. Casarini, On general features of warm dark matter with reduced relativistic gas. arXiv: 1706.08595

25. J.C. Fabris, I.L. Shapiro, A.M. Velasquez-Toribio, Testing dark matter warmness and quantity via the reduced relativistic gas model. Phys. Rev. D 85, 023506 (2012). arXiv:1105.2275

26. F. Jüttner, Das Maxwellsche Gesetz der Geschwindigkeit sverteilung in der Relativtheorie. Ann. der Phys. Bd 116, 145 (1911)

27. W. Pauli, Theory of Relativity (Dover, New York, 1981)

28. R. Hakim, Introduction to Relativistic Statistical Mechanics (World Scientific Publishing Co. Pte. Ltd., London, 2011)

29. Y.B. Zeldovich, A hypothesis, unifying the structure and the entropy of the universe. Mon. Not. R. Astron. Soc. 160, 1P (1972)

30. E.M. Lifshitz, I.M. Khalatnikov, Investigations in relativistic cosmology. Adv. Phys. 12, 185 (1963)

31. Y.B. Zeldovich, I.D. Novikov, Relativistic Astrophysics. Vol. 2. The Structure and Evolution of the Universe (Chicago University Press, Chicago, 1983)

32. E. Schucking, O. Heckmann, Newtonsche und Einsteinsche Kosmologie. Handbuch der Physik 53, 489 (1959)

33. Wolfram Research, Inc., Mathematica, Version 9.0. Champaign (2012)

34. I.M. Khalatnikov, A.Y. Kamenshchik, A generalization of the Heckmann-Schucking cosmological solution. Phys. Lett. B 553, 119 (2003). arXiv:gr-qc/0301022

35. J.C. Fabris, A.M. Velasquez-Toribio, W. Zimdahl, I.L. Shapiro, Interacting photon-baryon fluid, warm dark matter and the first acoustic peak. Eur. Phys. J. C 74, 2968 (2014). arXiv:1312.1937

36. G.L. Comer, P. Peter, N. Andersson, Multi-fluid cosmology: an illustration of fundamental principles. Phys. Rev. D 85, 103006 (2012). arXiv:1111.5043

37. G.L. Comer, P. Peter, N. Andersson, Cosmological two-stream instability. Phys. Lett. B 715, 289 (2012). arXiv:1111.5607

38. D.Müller, A. Ricciardone, A.A. Starobinsky, A. Toporensky, Anisotropic cosmological solutions in $R+R^{2}$ gravity. arXiv: 1710.08753

39. A.Y. Kamenshchik, E.O. Pozdeeva, A.A. Starobinsky, A. Tronconi, G. Venturi, S.Y. Vernov, Induced gravity, and minimally and conformally coupled scalar fields in Bianchi-I cosmological models. arXiv: 1710.02681

40. A. Ashtekar, E. Wilson-Ewing, Loop quantum cosmology of Bianchi I models. Phys. Rev. D 79, 083535 (2009). arXiv:0903.3397 